\title{
Expériences de fin du monde : un jansénisme en convulsion, un calvinisme en prophétie
}

Daniel Vidal

\section{(2) OpenEdition}

1 Journals

Édition électronique

URL : http://journals.openedition.org/assr/20725

DOI : $10.4000 /$ assr.20725

ISSN : $1777-5825$

Éditeur

Éditions de l'EHESS

Édition imprimée

Date de publication : 1 juin 2001

Pagination : 21-37

ISBN : 2-222-96704-X

ISSN : 0335-5985

\section{Référence électronique}

Daniel Vidal, «Expériences de fin du monde : un jansénisme en convulsion, un calvinisme en prophétie ", Archives de sciences sociales des religions [En ligne], 114 | avril-juin 2001, mis en ligne le 19 août 2009, consulté le 02 mai 2019. URL : http://journals.openedition.org/assr/20725; DOI : 10.4000/ assr.20725

Ce document a été généré automatiquement le 2 mai 2019.

() Archives de sciences sociales des religions 


\title{
Expériences de fin du monde : un jansénisme en convulsion, un calvinisme en prophétie
}

\author{
Daniel Vidal
}

\section{RÉFÉRENCE}

CHANTIN (Jean-Pierre), Le Jansénisme, Paris, Cerf, 1996, 126 p. (coll. «Bref »)

CHANTIN (Jean-Pierre), Les Amis de l'‘uvre de la Vérité - jansénisme, miracles et fin du monde au XIXe siècle, Lyon, Presses Universitaires de Lyon, 1998, 186 p.

CHABROL (Jean-Paul), Élie Marion, le vagabond de Dieu (1678-1713), Prophétisme et millénarisme protestants en Europe à l'aube des Lumières, Aix-en-Provence, Édisud, 1999, 272 p.

1 Au commencement, le verbe du malheur : une foi combattue, une culture stigmatisée au risque de sa perte absolue, des sites à forte charge symbolique anéantis, des territoires de religion dévastés. Nulle histoire, sans doute, qui ne soit tissue de ces violences et fureurs. Mais nulle, aussi bien, qui ne décide par là même de toute une raison, patiente ou explosive, de refus radical. Au seuil de notre histoire contemporaine, deux rigoureuses institutions de religion connurent oppressions à la mesure de leur emprise culturelle et cultuelle, de leur science de Dieu, et de leurs défis politiques. Jansénisme et calvinisme furent proies de tous les tourments des siècles où ils s'épandirent. Et inventèrent, au sein même des allégeances religieuses dont ils ne cessèrent de témoigner, des épreuves singulières de corps et de verbe, pour tenter non seulement de simplement survivre au monde qui les accablait, mais aussi bien d'y installer des ferments de dissidence sans recours. De là les convulsions jansénistes à Paris et en toutes provinces, et les prophéties calvinistes en terre languedocienne, puis en exil européen. Trois ouvrages affinent la connaissance de ces épreuves, fondées sur un argumentaire où se conjoignent miracles, convulsions, théorème de fin du monde, millénarisme et prophéties. Du jansénisme, J.-P. 
Chantin rappelle l'avènement et l'ample déploiement au vif de l'Église de France. Et ses recompositions au fil des décennies et des régions, jusqu'au cœur du XIX ${ }^{e}$ siècle en l'espace lyonnais. J.-P. Chabrol reformule le paysage du prophétisme cévenol après le soulèvement camisard, à partir de la figure emblématique d'Élie Marion et ses voyages d'une ville d'Europe à l'autre. On pressent que les ouvrages de Chantin et Chabrol offrent aux sciences sociales une exemplaire contribution à l'intelligence des opérations qui travaillent le siècle des Lumières de son aube à sa fin.

Dans son Jansénisme, J.-P. Chantin tente de condenser en un peu plus d'une centaine de pages l'essentiel de ce qu'il convient de savoir sur le mouvement de Port-Royal: les exigences de collections de brève synthèse sont rudes. Comment y parvenir, quand l'A. convient, après R. Taveneaux et A. Gazier, qu'il faut traiter du jansénisme au pluriel, si l'on veut être au plus près de l'histoire. Jean Orcibal avait à son tour distingué augustinisme, port-royalisme, grand jansénisme du XVII ${ }^{\mathrm{e}}$, richérisme, quesnellisme, selon les figures organisatrices de cette mouvance de religion. J.-P. Chantin y réussit au mieux, au prix sans doute d'une composition très ramassée du répertoire historique, qui peut déconcerter le lecteur par sa densité. Prenant le parti d'une "vision globale, mais nécessairement succincte, de cette 'énigme historique' ", l'A. dessine cependant une fresque à grands traits, tous pour l'essentiel pertinents et savamment présentés, de cette vaste entreprise de refondation religieuse aux confins de l'hétérodoxie et d'un radicalisme fondamentaliste. Périodisation sans surprise, mais sans lacunes. L'ère des grands initiateurs, porteurs d'enjeux théologiques décisifs : débat sur la grâce, la nature déchue de l'homme, l'absolue contrition pour une parfaite communion, le nécessaire retour aux pratiques des premiers temps chrétiens, etc. Voici le "premier jansénisme». Des personnages emblématiques lui donnent toute sa splendeur et sa résonance, noms entrés en l'histoire comme figures essentielles, et qui font désormais partie de notre patrimoine culturel : Saint-Cyran, Antoine Arnauld et son prestigieux lignage, Nicole, Pascal. Tous inscrivant une profonde crise au cœur de l'Église romaine : en 1654, la bulle Cum occasione dénonce cinq propositions portées au compte de Jansénius, et le clergé exige la signature d'un formulaire condamnant l'Augustinus de l'évêque d'Ypres. À ce grand moment de vertu religieuse et de dissidence culturelle succède une autre configuration, plus politique et " populaire ». Le rasement de Port-Royal à partir de 1711, la bulle Unigenitus condamnant en 1713 cent une thèses de Quesnel, font basculer le jansénisme en une clandestinité fiévreuse. Appelants, réappelants, opposés à l'interdit pontifical: toute une frange du bas clergé et des hauteurs de l'institution ecclésiale, passent en insoumission, tandis que les héritiers de Port-Royal forment terrain de révolte de corps et d'âme. Un relais politique : le Parlement, largement habité de jansénisme, opposé à l'absolutisme monarchique. Au sein de l'Église, de nombreux ordres et congrégations à leur tour entrés en inquiétude. J.-P. Chantin évoque les batailles frontales ou feutrées, jusqu'à la vaste entreprise des convulsions, en laquelle il note la naissance d'un «merveilleux » janséniste : temps des miracles et des transes sur le tombeau du diacre Pâris, qui durera jusqu'à la fin du siècle, porteur de défis politiques, annonciateur d'ébranlements du siècle ${ }^{1}$. L'A. propose quelques critères pour démêler la question redoutable du rôle, direct ou indirect, celui-ci plus vraisemblable, du convulsionnisme janséniste, figuriste ou plus apaisé, dans l'entrée de la société française en Révolution. Question majeure, réponse nécessairement équivoque. Ralliement à la constitution civile du clergé pour certains, opposition absolue pour d'autres. De là des sécessions en chaîne, des «sectes » à foison - tout un paysage d'«agonie » qu'aggravent la signature du 
Concordat, la naissance des « petites églises » segmentées ${ }^{2}$, etc. Demeure la marque d'une " société » janséniste, que l'A. évoque à travers la figure du prêtre, en qui se concentrent la volonté de rénovation des mœurs, l'aspiration à fonder le 'nouvel homme', l'exigence de retrait du monde, l'argument de pénitence, et, contre le libéralisme protestant en matière de prêt à intérêt, la détestation de l'usure. Mais l'ensemble de ces théorèmes ne peuvent organiser sur le long terme la pratique d'une société civile en train de se constituer, non plus que résister à la nouvelle hégémonie de Rome et le triomphe d'une piété ultramontaine. Le gallicanisme œuvré par le jansénisme du Grand Siècle et du siècle des Lumières se délite. Mais on sait que le meilleur de la culture européenne aura, jusqu'à nos jours, puisé au plus profond de cette éthique radicale, les raisons de sa dignité.

3 Si l'on connaît en profondeur cette grande geste nationale du jansénisme, ses tenants théologiques, ses aboutissants politiques et culturels, il est des zones cependant de plus grande obscurité. Soit que, du fait de leur inscription nécessairement locale et provinciale, elles n'aient pas, pour l'essentiel, accédé à la légitimité d'objet d'analyse historienne, soit qu'elles n'aient pas paru ajouter quelque chiffre nouveau à l'argumentaire central. Il y a là méconnaissance dommageable à l'intelligence de l'entier héritage de Port-Royal. Toute une part de celui-ci concerne le tournant des $\mathrm{XVIII}^{\mathrm{e}}$ et XIX ${ }^{\mathrm{e}}$ siècles, qui voit la lente, et obsédante, extinction de ce vaste mouvement d'insoumission religieuse : le jansénisme n'en finit pas de jeter ses ultimes feux jusqu'au plein du XIXe, après deux siècles, on l'a vu, de présence au plus intime de l'histoire de l'Église, et de la nation. On sait sa prime histoire, et déjà l'on soupçonnait une quête inachevable de ses raisons, chaque moment qui paraissait faire décision renvoyant à une configuration antérieure de sens. Jansénius, bien sûr, et son Augustinus. Qui est écho de saint Thomas, lui-même argumentant le théorème d'Augustin, saint Docteur de la grâce. Drame des origines: elles habitent la nuit des temps - noire matrice de tout événement capable de décider d'un monde. On peut alors, au prix de cette remontée aux sources dérobées, identifier des périodes de plus grande crue : ampleur soudain prise par tel élément de doctrine dans un débat où se conjuguent enjeux théoriques, institutionnels, politiques. Ainsi de la longue guerre sans merci qui nous occupe ici, opposants tenants de la grâce efficace et de la prédestination gratuite, à ceux de la primauté des œuvres dans l'assignation à salut ; ou partisans de la seule attrition dans la pratique de la communion, à ces hommes de haute vigilance, qui exigent absolue contrition et aveu radical de son péché pour accéder au corps et au sang de son dieu. Encore fallait-il traquer la première lueur d'où put ainsi jaillir, dès l'aube du XVII ${ }^{e}$, la grande lumière de Port-Royal. Le jansénisme sera dès lors en permanence requis, de par sa propre obligation éthique ou par contrainte externe, de faire la preuve de sa légitimité doctrinale. Et conduit par là à toujours reprendre le cours de ses héritages, par quoi le plus lointain passé ne cesse d'affluer dans un présent de plus forte urgence.

4 Le jansénisme finissant, dont J.-P. Chantin propose en son ouvrage sur les Amis de l'Euvre un éclairage minutieux et une mise en place fondée sur des ressources archivistiques pour la première fois, me semble-t-il, ainsi articulées, conduit l'historien aux mêmes seuils d'indécidabilité que la quête des origines. On a nommé cette mortesaison du jansénisme, son agonie ${ }^{3}$. Pouvait-on proposer plus exacte définition! Mais à condition de bien entendre ce vocable : non point un temps très court âprement disputé à la mort, qui étreint déjà le vif, mais une infinie durée qui borde cette mort sans s'y soumettre. Il est certes des agonies d'heure dernière, par quoi s'achèvent brusquement les paysages les plus nets. Mais il en est qui ne connaissent pas de fin, sauf à valoir celle 
du monde même. Pascal, ici, de toute nécessité: Jésus «en agonie jusqu'à la fin du monde : il ne faut pas dormir pendant ce temps-là »4. Le jansénisme pouvait-il, en état d'extrême abandon, finir au monde, lui qui n'avait cessé, au plus haut de sa névralgie, d'en clamer la fin proche, reportant l'échéance comme pour ne l'atteindre jamais, et jubiler de se savoir ainsi maître d'un jeu de rare complexité ? ${ }^{5}$ Voici la plus tragique des agonies, qui n'en finit pas de surseoir à sa fin. Le jansénisme ainsi demeure en état de veille, au seuil d'une mort qu'il ne côtoie que sur un mode asymptotique. Et de même que les premiers temps du jansénisme furent temps de retournement vers les grandes figures du passé, et leur exaltation jusqu'à la plus exigeante tension, de même tous gens habités de cette tension même ne cessent, des dernières décennies du XVIII ${ }^{e}$ jusqu'au milieu du $\mathrm{XIX}^{\mathrm{e}}$, de reconduire en leur exactitude les théorèmes fondateurs touchant l'impératif de la grâce, le néant de la créature, et les pratiques illuminantes des miracles et convulsions. Mais ce temps qui, à vrai dire, semble ne plus passer, puisque il est temps de reproduction à l'infini des grandes stratégies du sacré, est pleinement vecteur d'historicité ardente, brisant les complicités séculaires, et disposant au cœur du mouvement janséniste, des principes de sécession. Mais là encore, un même scénario conjoint l'aurore du jansénisme et son crépuscule - on sait les débats sur le "merveilleux ", contemporains des miracles de Port-Royal ${ }^{6}$, et, au XVIII ${ }^{e}$, ceux qui opposèrent, toute référence figuriste partagée, les partisans des convulsions et leurs adversaires résolus ${ }^{7}$.

Lyon offre, en une pureté de cristal, la plus complète leçon d'histoire du jansénisme "tardif ». L'analyse magistrale de L. Trénard ${ }^{8}$ permet de comprendre dans quelles conditions culturelles et sociopolitiques ont émergé en amont et en aval de la césure de quatre-vingt-neuf les réseaux de sociabilité hétérodoxe, loges, écoles de pensée dissidente, illuminisme et prophéties, et tout ce qui énonce et travaille le champ politique à partir d'une mise à nu et au net d'enjeux sociaux et symboliques opérant à même l'instance de religion. J.-P. Chantin assoit avec bonheur sur cette œuvre princeps son enquête sur les multiples configurations jansénistes qui constituent, en cette période où un siècle bascule sur l'autre, l'héritage de Port-Royal en Forez, régions lyonnaise et stéphanoise. État des lieux guidé par le souci constant de ne laisser dans l'ombre aucune "Église ", aucune "société » d'Amis de l'œeuvre, qui foisonnent alors. L'ÆEuvre: l'accomplissement de la Vérité, en ce qu'elle porte témoignage de la désolation des fidèles dans la corruption généralisée du monde, et l'assurance que seule la fidélité aux premiers temps de l'Eglise peut autoriser une éternité de salut. CEuvre que magnifient miracles et convulsions, comme il en alla cent ans plus tôt dans la mouvance de Saint-Médard. Pour mieux comprendre que cette CEuvre ait ici connu un tel épanouissement d'arrière-saison, l'A. rappelle le rôle des conducteurs d'âmes, dont Mgr de Montazet, de 1758 à 1788 archevêque de Lyon, favorable aux thèses port-royalistes, la marginalité ultérieure des groupes concernés, qui induisent chez eux des conduites de résistance et une intransigeance doctrinale qui ancrent prophéties, attentes millénaristes, convulsions et secours, sur des terres d'incertitude : longuement éprouvées par la réforme tridentine, elles connaissent désormais un moindre effort de conversion, par l'« essoufflement " peu à peu de cette « reconquête ». J.-P. Chantin désigne ainsi les opportunités qui s'ouvraient aux héritiers de Port-Royal pour attester la permanence de leur "Vérité », contre les abandons d'une Église soumise à Rome, et pour la singularité de l'expérience de la grâce. Je reviendrai sur ce thème de la permanence, qui constitue l'une des questions majeures posées par l'A. 
6 Mais il faut s'attarder un instant sur cette " originalité lyonnaise », qui intrigue en effet tout analyste. Il me semble que les différents recours explicatifs qu'avance J.-P. Chantin prennent tout leur sens si l'on accepte de lire dans le travail de convulsions, l'œuvre miraculiste, les prophéties d'apocalypse, les attentes millénaristes, non point seulement une protestation contre un ordre doctrinal figé dans sa volonté de puissance et de raison, pas plus que le seul rappel pathétique aux fondements de la foi en ses merveilles même, et l'imitation de la crucifixion pour venir à l'image de son dieu. Mais une opération de connaissance radicale des corps et, pour reprendre l'expression de M. Bergamo, de «l'anatomie de l'âme »'. C'est par là que " l'CEuvre de la Vérité » me paraît valoir comme carte maîtresse circulant en milieu lyonnais dans la période qui nous occupe ici. Soit les convulsions: si l'on récuse l'interprétation en termes "médicaux", telle qu'elle fut avancée au XVIII ${ }^{\mathrm{e} 10}$, et que Sainte-Beuve reconduisit sans autre forme de procès, liant les convulsions ultimes aux miracles jansénistes du Grand Siècle ${ }^{11}$, un nouveau modèle interprétatif se dessine. Lire les miracles, c'est aussitôt entrer en une économie symbolique qui, par corps malade projeté au centre de la scène d'histoire, exhibe le mal comme condition du rapport social : il n'est pas de miracle qui ne soit affaire et raison de société. Si le miracle est bien ce qui capte la vision d'autrui, et le fait pénétrer dans la logique même de son énonciation, c'est pour signifier cette " vérité », que les jansénistes auront portée à son extrême conséquence : le mal du corps privé ne connaît son miracle que si ce mal circule dans ce que l'on nommerait aujourd'hui l'espace public, où il fait événement en permanence fondateur, et donc en permanence scandaleux. Car il est en effet un scandale dans cette entreprise de réamorçage sans cesse de la socialité, qui laisse entendre que rien jamais n'est si fragile, et précaire, qu'un lien social qui se donne pour immédiatement donné.

$7 \mathrm{Au}$ principe interlocutoire $\mathrm{du}$ miracle répond le principe organisateur du schème convulsif. On n'entre en état de spasme qu'au pluriel. Il y faut non seulement une société alentour, qui fasse structure d'accueil et de légitimation. Il y faut aussi une circulation du spasme en cette " assistance ", qui dès lors est à part entière requise de se conformer à sa logique. La transe d'un corps à l'autre circule: le spasme est monnaie symbolique qui entre en un procès d'échange où ce qui de l'un à l'autre se donne et se rend œuvre à la raréfaction du référent religieux : un corps en convulsion est corps offert à la vue et au savoir du siècle. Miracles et corps de spasme sont modalités de connaissance: ce qui compose ce savoir, tout au long du XVIII ${ }^{e}$, et en cette première moitié du XIXe siècle, se dit maladie, malédiction - le mal en tous ses états. L'œuvre capitale du jansénisme est d'avoir fait basculer le mal comme attestation de faillite au cœur de l'Église, en lieu et figure de connaissance, et son passage obligé12. Cette articulation entre symbolique figuriste et épistémologie qui opère à la destitution la plus radicale des référentiels, n'autorise pas seulement l'irruption du corps janséniste comme corpus du savoir au plein $\mathrm{du}$ siècle des Lumières. Elle permet, me semble-t-il, de comprendre comment convulsionnaires et miraculistes de toute " société ", «Église » ou " secte » jansénisante, quelle que soit leur allégeance circonstancielle, s'inscrivent sans difficulté dans le tissu culturel de Lyon. Je prendrai un seul exemple. Claude-François Desfours de la Genetière (1757-1819), miraculiste. anticoncordataire, acteur majeur dans l'histoire de la « Petite Église » de Lyon, qu'analyse en détail J.-P. Chantin. Desfours est oratorien, héritier passionné de Port-Royal, et d'une fortune paternelle qui lui permet de soutenir les fragments dispersés des fidèles de Mâcon, Saint-Étienne et Lyon. Fidèle à l'enseignement du dominicain Bernard Lambert, de vingt ans son aîné (1738-1813), qui avait bénéficié de 
la protection de l'archevêque de Lyon, s'était opposé à la constitution civile du clergé, après avoir publié en 1786-1787 un ouvrage favorable aux convulsions à secours, les plus sacrificielles, et de plus haut sacrilège pour les dévots ${ }^{13}$. Il s'était également engagé dans le débat complexe prônant le "retour des juifs", qui s'entend comme conversion à rebours de l'Église en une allégeance première à la loi mosaïque. Sur tous ces points, Desfours témoignera d'une stricte observance. J.-P. Chantin note qu'il envisagera de passer alliance avec une femme "de la communauté juive de Lyon", qui eut pu ainsi nouer liens précieux avec Félicité Boussin, alias sœur Isaac, dont j’ai traité par ailleurs. On le sait à l'évidence gallican, convulsionniste, miraculiste : le voici donc en terre lyonnaise, répondant à tous les critères de la singularité janséniste. Mais Desfours ne donne pas dans la seule inquiétude théologique, et la seule quête du dieu caché. Ou, plus exactement, cet argumentaire tragique entre en exacte consonance avec le siècle et ses mises à l'épreuve du sens des choses et de leur loi. C. Latreille rappelle qu'il fut éminent en l'art de la mathématique, des " qualités et efficiences des fluides ${ }^{14}$. Homme de spasme en tant que celui-ci porte connaissance rigoureuse du corps, au prix de la plus rude de ses " questions »; homme de savoir en tant que ce savoir est mise en question du réel.

C'est cette conjonction de raisons épistémologiques qui va, pour l'essentiel, décider de la rencontre à Lyon et sa région des réseaux jansénistes des derniers temps, des multiples vagabondages de l'esprit, de l'effervescence de constructions utopiques, et de mesures du monde. Mesure et démesure: avers et revers d'un même ensemble culturel, toujours allant de pair, catégories l'une à l'autre engrenées - Lyon, ou la cité lue comme palindrome. L. Trénard a longuement analysé la conjonction des argumentaires : les Amis de l'đEuvre de la Vérité en sont un exemple privilégié. Et c'est l'extrême intérêt de l'ouvrage de J.-P. Chantin, que de nous faire entrer au plus intime de ces groupes sur euxmêmes clos, et cependant ouverts à tous les vents de l'esprit le plus au large, et à l'histoire qui décide au coup par coup sa route et ses tourments. De la Révolution considérée comme la Grande Convulsion rédemptrice et punitive, vaste entreprise sacrificielle rétablissant en une forme de jugement dernier la Vérité des fidèles contre la corruption d'une Église sans aveu, à la ligne de fracture qui va traverser les Amis de l'Euvre face aux choix politiques ultérieurs - constitution civile du clergé, puis Concordat -, les concrétions jansénistes font présence active à même le champ politique. Chantin examine en détail les différentes étapes et modalités des divergences qui vont progressivement s'insinuer dans les Églises «dissidentes». Toutes se réclamant de l'œuvre des convulsions, elles vont connaître des destins opposés. Héritières de l'oratorien Pinel, « un des oracles des convulsionnaires à Paris et Saumur ", c'est à partir du lieu politique qu'elles entrent en conflit. J'y reviendrai. Mais ce lieu fut de tout temps l'un des enjeux majeurs du jansénisme. On sait le rôle des relais parlementaires au XVIII ${ }^{\mathrm{e}}$ siècle. Du sein de l'espace convulsif, cependant, avait été énoncée, parce que désirée, la grande émotion de quatre-vingt-neuf. J'avais relevé, au fil des décennies 1740-1780, les prophéties de la juste colère de Dieu et du retournement du monde, qui disaient la proche fin de ce monde ${ }^{15}$. J'en rappelle quelques-unes. Sœur Colombe, en 1742, annonçait cette convulsion de colère éclatant «non sur la France seule, mais sur toutes les monarchies, puisque toutes ont trempé leurs mains dans l'iniquité $»^{16}$. Sœur Dorothée, en 1750 : «Monarque impur, tes jours sont comptés. Tu périras sous ton sceptre, toi et ta courtisane serez frappés d'une mort bien tragique ", tous emportés "par le courant des eaux terribles $»^{17}$. Sœur Esther Perpétue, 1773 : «C'est bientôt, c'est bientôt, le tonnerre grondera... Une émeute terrible surviendra et le sang coulera en abondance. Vous me faites voir une désolation si grande et si universelle, que tout sera saisi et consterné $»^{18}$. 
Autre sœur Esther, janvier de la même année : «Et toi, Roi de la terre, qui d'un œil altier regardes périr ta nation, tremble pour toi ", car ce sont désormais années "de sang, de feu et d'eau $»^{19}$. «Je ne vois que sang répandu. Tout sera culbuté, Montmartre et les bâtiments renversés, Paris rasé » crie Félicité Lefranc en $1782^{20}$. Avril 1787, sœur Aile en convulsion : «Il faudra que la Révolution se fasse... Je ne vois qu'embuscade, je ne vois que précipice, le sang coule à l'entour de moi, j'entends le bruit des armes, le palais du roi est balayé, sa couronne lui est ôtée. Je me trouve enfoncée dans ce puits d'abîme, au milieu d'une si grande révolution $»^{21}$. Convulsionnaires proférant mille prophéties porteuses de mille malheurs, disant ce qui doit advenir parce que ce malheur multiplié est déjà advenu en leur histoire. Comme le rappelle J.-P. Chantin, le père Pinel, inspirateur de Claude Desfours, avait publié dès 1749 l'Horoscope des tems ou Conjectures sur l'avenir fondées sur les Saintes Écritures et sur de nouvelles révélations. Près d'un demi-siècle plus tard, Desfours lui-même publie en 1792 un Recueil de prédictions intéressantes faites depuis 1733, et, au début du XIX ${ }^{\mathrm{e}}$, paraît un Précis d'annonces des événements et révolutions temporelles et spirituelles qui doivent s'opérer dans toute la gentilité mais plus particulièrement en France ${ }^{22}$. Entre Pinel et Desfours, la Révolution. Entre le Recueil et le Précis, la constitution civile du clergé et le Concordat. Les convulsions scandent l'histoire au plus près de son déroulement: les gens du spasme n'énoncent tant de prophéties, que parce qu'ils font présence vive au cœur de leur société et de ses émotions.

9 Les «sectes" jansénistes du lyonnais ne sont pas seulement héritières des énoncés dévastateurs des générations précédentes. Elles légitiment cet héritage par le seul acte capable de rendre compte de la permanence de l'Cuvre: la convulsion. Non pas une assemblée du spasme comme il s'en tint des milliers tout au long du XVIII ${ }^{e}$, mais une séance de grands secours, emblématique des souffrances du temps, et reconduisant la plus haute tension à quoi puisse atteindre une décision de consumation des temps et d'accomplissement du corps glorieux. Ce secours : la crucifixion. Il s'en fit de multiples en ce siècle. Mais à Fareins, à peu de distance de Lyon, en 1787, le crucifiement d'une «prophétesse » eut valeur de refondation. Car telle est la logique d'un héritage : il ne perdure que si, le temps venu, recommencé. Et recommencé en sa plus extrême exigence, à quoi tout aboutit, et par quoi tout advient. Si la crucifixion de Fareins marque bien, ainsi que le note J.-P. Chantin, la «relève» par la province du mouvement convulsionnaire parisien qui va s'affaiblissant, elle témoigne aussi d'une radicalisation de l'événement du spasme: il n'est sans doute d'héritage bien conçu que dans la mise en excès des enjeux qui le composent. Je ne reviens pas sur les enjeux proprement épistémologiques, liés à la transfiguration du corps vivant en corpus de connaissance, après qu'un tel corpus se fut constitué quant au cadavre. Mais cet excès du spasme ouvre décisivement la voie à un engagement au comble du principe politique. Républicain, on fera, en écho de Sade, encore un effort pour l'être en son excès. Fidèle malgré tout à un Ancien Régime que l'on désirait cependant aboli, on refusera la prestation de serment au risque de la guillotine ${ }^{23}$, et la nouvelle configuration ecclésiastique imposée par le Concordat. Sans doute existe-t-il un parti médian, comme il en fut au siècle précédent, aménageur de compromis, rebelle à tout choix extrême ${ }^{24}$. J.P Chantin l'identifie avec précision dans les années qui nous concernent ici : ce sont les "communicants", plus aisément ralliés à l'Église au cours du XIX ${ }^{\mathrm{e}}$, sans pour autant renier l'essentiel de l'héritage port-royaliste, mais le vivant comme expérience intérieure plus que de façon ostentatoire. Les deux versants opposés de l'œEuvre, cependant, me semblent, en accord avec les données présentées par l'A., et celles que j'avais analysées par ailleurs, plus proches de la source vive des convulsions, puisqu'ils en ont refondé la vocation. A Fareins, 
tout se passe dans la mouvance et sous le contrôle des frères Bonjour. Il est intéressant en effet de comprendre qu'ici se nouent pour plusieurs décennies l'épreuve de convulsion comme témoignage de la Vérité, et la passion de Révolution comme accomplissement de cette vérité dans le champ du politique. C'est avérer ainsi la double violence par quoi la " vérité » doit se frayer la plus étroite des portes pour advenir au plein du rapport social, et celle dont l'insoumission politique doit être porteuse pour advenir comme vérité dans l'histoire.

A ce titre, Claude Bonjour a su mettre en consonance son expérience convulsionniste et sa pratique politique : le voici en 1789 à Fareins, avec son frère François, après l'épisode de la crucifixion, qui leur valut prison et exil. Retour triomphal. Leurs prédications?: partage des terres, fin de la religion romaine, deuxième venue du Christ. Dans la «délibération» du 27 octobre 1789, ils proclament la nullité du droit de propriété, l'indépendance de la femme et de l'enfant, l'impossible état de péché des parfaits. Porte étroite du salut, mais large ouverture sur un monde bouleversé. Pour ce monde, toute la révolution qui convient: les fareinistes sympathisent avec la Terreur, et seront dès lors traqués lorsque viendront les temps de réaction. Pour cette nouvelle venue du Christ, la question du "retour" des juifs. J'ai analysé en d'autres lieux la complexité de ce théorème, qui organise l'argumentaire janséniste de longue date ${ }^{25}$. J.-P. Chantin me semble minorer cette catégorie nucléaire du jansénisme, qui connait en ces tournants de siècles, et spécialement dans les groupes du triangle Fareins-Saint-Étienne-Lyon, une exceptionnelle importance. L'ensemble des énoncés de la sœur Isaac, par exemple, de la fin du XVIII ${ }^{e}$ jusqu'au milieu du XIX ${ }^{e}$, forme une seule et même phrase concernant le peuple juif salvateur. La convulsionnaire ne se contente pas d'aller « réveiller » les tribus juives dans leur tombeau. Elle proclame leur nécessaire retour à eux-mêmes, à leur foi première, condition du salut d'une chrétienté déchue. Plus encore, elle métisse sa langue d'une théorie de vocables pseudo-hébraïques, pour signer sa présence au cœur de cette judaïté, dont elle vient, et disperser assez la parole de son dieu pour laisser advenir la sienne propre, garante de la nouvelle institution de foi ${ }^{26}$. Cas limite, sans doute, mais qui, du plus profond de l'espace des convulsions, réalise ainsi la logique du théorème. Du moins peut-on saisir ici l'un des éléments les plus significatifs de cette permanence qui retient si justement l'attention de J.-P. Chantin, par-delà les modulations singulières des référents centraux.

11 Il est encore, en cet ouvrage d'historien, des précisions essentielles. Ainsi de l'importance, déjà notée par l'A. dans son Jansénisme, du prêtre et directeur de conscience dans la continuité spirituelle et culturelle des cercles jansénistes ultimes, avec les énoncés majeurs du jansénisme. Domine ici, en ce premier tiers du XIXe, la belle et calme figure de François Jacquemont, qui ne rejoignit ni « béguins » de Saint-Jean Bonnefonds, ni Église anticoncordataire de Lyon, mais avait répandu les ouvrages port-royalistes en sa paroisse de Saint-Médard en Forez. Un siècle le sépare de François de Pâris, diacre de SaintMédard, mais ces années semblent ne point compter : le temps paraît en lui-même se clore, en ce nom de si haute résonance. Jacquemont meurt en 1835. Miracles et convulsions avaient trouvé en lui un défenseur sans complaisance, quelque distance qu'il fût amené à prendre, rappelle J.-P.Chantin, à l'égard d'improbables prophéties. Il fut à coup sûr modèle parmi les modèles pour une cohorte de prêtres attentifs à perpétuer les principes du jansénisme. Mais à côté, ou à défaut, de ces prêtres fidèles à leur foi profanée, J.-P. Chantin relève le rôle prépondérant des laïcs dans le maintien du portroyalisme en ces zones d'insoumission politique et religieuse. Du port-royalisme en tous 
ses états : convulsionniste, il va de soi ; miraculant aussi bien. Mais, plus encore, en son éthique même. Quand les cadres « légitimes » de l'institution d'Église se dérobent, ou sont réduits au silence, affluent en l'histoire ces hommes et femmes qui, hors de toute fonction dûment consacrée, assurent l'édification des fidèles « en marge de l'Eglise ». Laïcs au sein de « sectes » dissidentes, ils vont alors détenir le redoutable pouvoir de dire une nouvelle fois, celle-ci décisive, l'ancienne loi, et les conditions de son accomplissement quotidien. On comprend mieux alors, avec l'A., pourquoi la famille occupe le lieu privilégié de la mémoire janséniste. Il n'est ici nulle posture de repli, moins encore de déni d'autrui. Mais la condition de la réassurance de la loi en la seule modalité qui convienne alors, l'impératif éthique.

Et c'est pourquoi, sans doute, pour cette mise à nu des fondements du sacré, seul un peuple profane peut être requis. Il est en effet, dans l'urgence de son histoire, contemporain de la nouvelle donne qui s'est mise en place peu à peu au cours du siècle précédent, et qui désormais s'épand en toute expérience sociale, où se conjoignent science $\mathrm{du}$ malheur et appropriation personnelle de référents désenchantés. L'argumentaire janséniste s'exalte alors en éthique scrupuleuse, bien au-delà des clivages sociaux que l'on a pu être parfois tenté d'habiliter. Il apparait que l'ensemble de la structure sociale est de haut en bas traversé par cette fêlure janséniste qui n'en finit pas de l'inquiéter. Si J.-P. Chantin note en l'espace lyonnais le rôle des négociants dans l'émergence des Églises séparées, c'est pour relever aussitôt la « clientèle » des " masses déracinées ». Cette véritable déhiscence verticale, que j'avais analysée aussi bien dans la "société des miracles » au temps du Grand jansénisme qu'au long du XVIII , et que l'on retrouve en ces fragments éclatés et cependant solidaires qu'étudie J.P. Chantin, interdit de raisonner en termes de stratification sociale. Bien plutôt doit-on considérer cette échappée belle hors des rapports de distinction et de pouvoir, comme la plus grande chance pour le déploiement d'une éthique rigoureuse et, en effet, laïque, forcément laïque. L'Eglise instituée peut alors fort bien réabsorber ces éléments depuis plus de deux siècles en rupture d'obédience, ainsi que le constate Chantin: elle ne fait que prendre en charge une insoumission éthique qui ne cessera de la perdre de questions. Car ces cercles de jansénistes, s'ils demeurent fidèles aux leçons de Port-Royal, ne peuvent pleinement les vivre que comme douleur attachée à un impossible retour vers un paysage immémorial, à la fois intimement présent au foyer de la mémoire, et hors de toute remémoration. Nostalgie sans doute, mais, pour reprendre l'expression de Baudelaire, «nostalgie du pays qu'on ignore». Subsiste alors, de l'œuvre immense de Port-Royal, cette vocation à penser le monde comme impossiblement habitable, et cependant le seul où l'on puisse en vérité demeurer. Car cette pensée vaut fondement de l'éthique, par quoi le malheur est destin en même temps que catégorie centrale de l'espace intérieur où s'organisent les instruments de la responsabilité personnelle.

Port-Royal des Champs est rasé en 1710. L'année suivante, le cimetière de l'abbaye est dévasté : cadavres déchiquetés, hachés, transportés «au tas commun (..), corps demi pourris ou même sanglants, jetés ainsi pêle-mêle les uns sur les autres ». Le chroniqueur dit que ce fut l'« abomination de la désolation » que de voir " des chiens amassés autour de quelques-uns de ces corps disloqués, et qui y cherchaient leur pâture $»^{27}$. Acte d'ultime sacrilège, après la chaîne d'interdits dont le jansénisme fut accablé. Les corps des Solitaires et des grands fondateurs furent dispersés : Pascal et Racine à St-Étienne du Mont, Saint-Cyran à St-Jacques du Haut Pas, Nicole à St-Médard, comme un signe des temps à venir. Lieux en effet d'où plus tard devait sourdre l'œuvre des miracles et 
convulsions, jusqu'à ce cœur du XIXe siècle que vient d'explorer J.-P. Chantin. En leur principe, ces désolations et ces ruines, ces censures et abaissements. Ces « abominations » sont monnaie courante en l'histoire. Et toutes le plus souvent décident, mais en sourdine, d'une longue suite de surrections de sujets venus à leur deuil comme à leur destinée, et s'y absorbant jusqu'à leur propre consomption. Pour en avérer la tragédie fervente, il suffirait, attentif à ce qui se trame sous les raisons pacifiées, et le normes recommencées, d'écouter les rumeurs du dehors du temps, de déchiffrer les emblèmes de la passion, et d'entendre paroles de prophètes. Car ils viennent, seuls ou infiniment multipliés, disant le malheur du monde abattu sur eux, et comment, en ce malheur même, vivre à contretemps et contre raison. Lorsque Port-Royal est rasé, pour l'essentiel l'insurrection camisarde en Languedoc calviniste est jugulée. Le pouvoir y avait mis le prix le plus exorbitant : brûlement des Cévennes, traque des insurgés, décapitations, galères, exils, ravages des terres et des consciences. Ces consciences depuis une génération avaient subi la plus irréparable sans doute des blessures : l'abjuration de leur foi, contrainte, ainsi, et humiliée. Les décennies précédant la révocation de l'Édit de Nantes avaient connu conversions forcées et, déjà, chasses aux hérétiques récalcitrants. Des pasteurs, conducteurs de fidèles pour certains, avaient résisté, d'autres ayant monnayé leur retour à l'Église de Rome. Les faits sont maintenant connus $^{28}$. Demeure cette floraison de prophètes, de tous âges, en toutes régions soumises aux dragonnades, et leur rôle majeur dans la conduite des hostilités. On ne prit pas les armes aussitôt, mais très vite vint le temps des « âmes ardentes ", pour reprendre l'expression de Paul Hasard, que rappelle J.P. Chabrol en son ouvrage sur Élie Marion et le prophétisme cévenol. Et ce temps dura près de trois décennies, porteur de prophéties en son Languedoc d'origine, puis en pays d'une Europe déjà irriguée de mille ferveurs lumineuses.

De ces théories de prophètes cévenols, à coup sûr l'un est figure majeure : Élie Marion, de Barre des Cévennes (1678-1713). J.-P. Chabrol analyse en détail les raisons et aboutissements de son aventure spirituelle, son engagement parmi les insurgés, sa décision prophétique, son parcours européen, sa mort en rade de Livourne, sur le chemin de Rome où il allait avec quelques inspirés du Refuge londonien "déclarer la parole ». Famille huguenote de longue date, lignée d'artisans se haussant au fil des générations jusqu'à un statut de "petite bourgeoisie » locale, sans prétention sans doute, mais sans complexe. Prise dans la tourmente des choix impossibles et des décisions à rebours des convictions intimes : le père d'Élie ainsi contraint de "servir » aux côtés des autorités, sans doute un temps très court - mais de quel poids ce temps-là pesa-t-il aux yeux du fils engagé en insurrection et prophétie? Il me semble, sans aucunement accorder importance majeure à cette « défaillance » du père, que, multipliée, elle participe de l'une des raisons de l'entrée massive, impatiente, du plus profond du peuple protestant en l'espace d'inspiration. Les inspirés, des Cévennes au Vivarais et au Castrais, sont contemporains des premières abjurations: ils donnent ainsi forme, et formule, à l'insoumission des consciences, en ces premiers temps du refus. Protestation qui ne s'insurge pas seulement contre les interdits de toutes sortes qui frappent les communautés réformées - temples détruits, cultes pourchassés, inhumations clandestines en sol huguenot. Mais qui dit aussi bien le vaste traumatisme provoqué par la faillite des pasteurs, la transgression des allégeances spirituelles, et l'hémorragie des sens qui s'y déployaient. De ce vide ainsi créé, la prophétie se fonde.

15 Lorsque vient le temps de l'insurrection, une génération est passée. Les premiers camisards assument ainsi, comme leur croix, une double culpabilité - d'être héritiers 
d'une foi déniée qui ne se perpétue que masquée et honteuse; d'être fils de ceux-là mêmes qui ont abjuré et les ont ainsi conduits, de force bien sûr, parfois de gré, sur les voies du reniement. Cris de repentance, clameurs de pénitence, forment d'emblée la double matrice de la prophétie. Mais en un second temps, qui concerne précisément la phase des énoncés proférés au Refuge, la même prophétie s'« accomplit » selon une autre pente : elle ajoute à cette passion d'amendements et de repentirs, le retournement sur soi du malheur accompli en l'histoire vécue, et la parole, comme une guerre, déclarée aux villes en sommeil. De la pénitence exigée aux fidèles en rupture de foi, à la prophétie de malheur - malheur pour soi, malheur pour les ennemis de la "vérité "-, Élie Marion apparait le héraut. J.-P. Chabrol examine avec une extrême précision les étapes du prophète sur les chemins de la science de prophétie, comme il en fut au siècle précédent de la «science » des saints en pays de catholicité traversés de passion mystique. Je dis 'science', car il ne suffit pas, il va de soi, de demeurer en l'espace de repentance pour faire prophétie. La prophétie n'est connaissance des temps à venir que d'être savoir déjà là d'un monde net comme tragédie, et toute cruauté. Alors peuvent se dire les temps d'apocalypse, d'autant plus porteurs de saccages ${ }^{29}$ qu'ils sont répercutés à l'ensemble des sites sociaux et symboliques que le travail de dieu institue travail de deuils. C'est en cette jonction d'une compétence à vouloir ce qui doit advenir, et d'une vocation à décider des neuves lois de l'esprit, que J.-P. Chabrol admirablement inscrit le prophétisme d'Élie Marion dans l'héritage des grandes traditions millénaristes et protestataires venues du fond d'une Réforme entrée en véhémence. Nulle recherche d'un historien français, jusqu'à cet ouvrage éclairant, méthodique, documenté, n'a mis au net, à travers la figure de Marion, cet ancrage décisif du prophétisme languedocien dans la longue histoire des espérances millénaristes antérieures, pour l'essentiel configurées par la Réforme, puis en ses marges et dissidences. Élie Marion prophète est homme présent à son histoire et à son monde : on sait, chez tout inspiré et mystique, cette absolue nécessité d'être pleinement de son temps, et donc partie prenante de ses tourmentes et ses rages. Marion, contre l'inclinaison de son père, mais de concert avec ses frères, prophétisera et participera aux actions de guerre, négociera redditions et exils, intermédiaire, dit J.-P. Chabrol, entre peuple et puissances. Les prophéties sont le dit de cette double inscription " civile » et "symbolique", leur permutation permanente, d'autant plus de facture "utopique" qu'elles parlent du seul réel effondré, et ne traitant des affaires du temps que pour mieux en livrer le chiffre de Dieu.

Il est en prophétie calviniste, note l'A., une mémoire vive et cependant immémoriale de l'histoire antérieure des signes divins. C'est le registre de cette mémoire qui est réactivé par les inspirés languedociens, aidés en cela par Jurieu, le théologien de Rotterdam, qui avait publié au lendemain de la Révocation L'accomplissement des prophéties sur la délivrance prochaine de l'Église ${ }^{30}$. Pierre Du Moulin, son aïeul, l'avait précédé sur la voie de ce déchiffrement spirituel et figuriste, en écrivant déjà son illustre Accomplissement des prophéties, et lorsque Marion entre en inspiration à son tour, il est à l'évidence débiteur de cette très riche histoire des symboliques de la puissance et présence réelle de Dieu dans cette histoire de toutes les fureurs. Les Avertissements prophétiques qu'il énonce au refuge londonien ${ }^{31}$ s'inscrivent dans cette "tradition » d'une temporalité réduite à un ultime spasme, quand tout se précipite vers l'acte crucifiant du jugement. Parmi tant d'autres énoncés : «Un temps est proche, il est court, il est à la porte ; il va arriver au premier jour (...). Mon enfant, je m'en vas mêler dans peu de jours, la joye avec la tristesse, l'angoisse avec les ris (...). Voici leur temps qui va tomber $»^{32}$. Ceci, encore: «Je m'en vais faire justice dans peu de jours. Que mon peuple se tienne pour averti, que je suis à la porte, que 
je suis à la veille, je te dis, de venir faire visite sur la terre.. Je m'en vais, dans peu de jours, donner l'accomplissement aux choses prédites depuis longtemps $»^{33}$. Cette précipitation du temps, cette veille, les compagnons inspirés de Marion, ces vigilants, au long de leur pérégrination européenne, ne cesseront de la clamer, écrivant ainsi une page de plus sur le grand livre des prophéties de l'imminence. J.-P. Chabrol note avec précision cette reprise, par l'inspiration languedocienne, de la tradition proprement réformée, ellemême, il va de soi, débitrice des figures fondatrices des millénarismes chrétiens, pour l'essentiel à base vétéro-testamentaire. Marion se situe ainsi au croisement d'un double défi, et porteur d'un double scandale, dont l'A. rappelle la fonction heuristique : mettre à nu la faute des pères et des conducteurs de conscience ; sur cette culpabilité, ce malheur survenu dans l'histoire réelle, fonder une prophétie de justice, qui vaut châtiment des ennemis de la foi, mais aussi bien du « peuple de Dieu » lui-même. Si bien que la prophétie s'accomplissant est porteuse des plus grands saccages.

Il fallait, à la suite de J.-P. Chabrol, rigoureusement penser ce métissage des deux grands énoncés prophétiques, l'un qui m'apparait fondé sur le paradigme de la faute, l'autre déployant l'argument millénariste jusqu'à sa raison utopique, pour comprendre l'écho multiplié que Marion et sa petite cohorte d'inspirés rencontrent à Londres et dans les pays du continent qu'ils vont traverser pendant sept ans. Ici commence en effet l'affaire des "French Prophets", cette "communauté " d'inspirés et d'« enthousiastes ", qui s'agrègent autour des exilés languedociens - Marion, Jean et Henriette Allut, Durand Fage, etc ${ }^{34}$. De même qu'en Cévennes les énoncés inspirés s'inscrivaient en un régime de prophéties que certains théologiens protestants avaient maintenues comme autant de "larmes amères » des années de guerre et de répression, de même en Angleterre ils entraient immédiatement en consonance avec "une nation saturée d'eschatologie", selon l'expression de F. Laplanche, que reprend J.-P. Chabrol ${ }^{35}$. Aux yeux des millénaristes anglais, et dans un contexte politique au début permissif, les inspirés cévenols apparaissaient, écrit l'A., comme « les derniers vestiges d'un puritanisme contestataire en quête d'une société idéale et pure, chrétienne et 'égalitaire' ». J.-P. Chabrol rappelle la très longue histoire de ces radicalismes religieux issus, pour s'en tenir aux bordures des temps annonciateurs de la Réforme anglaise, de Wycliff l'hérésiarque, le «Docteur Évangélique », et de l'insurrection des Lollards, par qui se formulèrent de façon irréversible les premières mises en question de Rome et de ses dogmes. Si cette dissidence majeure fut traversée de mysticisme et d'insoumission politique ${ }^{36}$, elle ouvrit la voie aux différents courants de l'apocalyptisme réformé, des millénarismes et messianismes d'où vinrent, aux temps modernes, quakers et philadelphiens, - ultimes adeptes d'une « interprétation littérale de l'Apocalypse », note l'A., et extrêmes contemporains des cévenols venus à Londres.

Il s'ensuit qu'en ce "pays des sectes $\aleph^{37}$, les camisards inspirés allaient pouvoir être prophètes d'exil, après l'avoir été en leur pays natal. L'A. conduit avec précision l'analyse de ce qu'il faut bien désormais nommer un mouvement religieux de vaste envergure, et souligne, en même temps que les héritages dont il procède comme d'une matrice de commun partage - anabaptistes, hussites, puritains, piétistes -, les harmoniques avec les réseaux contemporains - presbytériens, baptistes, congrégationalistes, etc. -, et ses influences au long terme sur des millénarismes et utopismes religieux dont notre temps demeure encore le témoin. L'itinéraire de Londres à Rome au travers d'une Europe qui se relève à peine des grands déchirements religieux des siècles précédents, est à la fois un marquage identitaire et, me semble-t-il, une aventure spirituelle mettant à nu les apories 
de cette assignation. Le petit groupe qui va et vient entre Londres, Amsterdam, la Prusse, Nuremberg, Stockholm, Prague, Hambourg, Smyrne, etc. pour achever ses périples à Rome, parcourt des espaces hautement symboliques : ici des pôles anabaptistes, ici encore des sites de haute mémoire hussite, là des régions où le piétisme commence à s'affirmer, et des zones où font encore rage des exigences de radicalité religieuse. Traverser ces espaces sacrés, c'est aussitôt provoquer des occasions de rencontre, pacifiques ou conflictuelles - mais qui pour l'essentiel renforcent la communauté des critères de l'inspiration, et les modalités de sa connaissance. Mais c'est aussi ajouter à la diaspora des révélations, un indice supplémentaire de variance : ainsi peuvent s'expliquer les poches d'hostilités auxquelles les prophètes cévenols vont se heurter parfois au cœur des cités où ils viennent déclarer des prophéties d'alarme, de saccage et d'éclair de lumière, comme un ultime sursaut de colère divine. Il importe surtout de saisir, avec J.-P. Chabrol, l'extrême densité des messages inspirés, capables assez de néant pour décider d'un désert d'après le malheur, et, au centre de ce désert, portant à incandescence des fragments d'énoncés bibliques seuls aptes à forcer des passages insoupçonnés dans la compacité des rhétoriques théologiennes. Aussi bien ces annonciateurs de jugements de Dieu habitentils des carrefours communs à des théories de « sectes » et groupements politico-religieux, d'autant plus dispensateurs de témoignages "divins », qu'ils sont présents à leur monde jusqu'en la plus fine pointe de sa durée.

19 À partir de ce petit noyau d'anciens camisards annonciateurs des temps de relèvement et de chute ${ }^{38}$, se constitue un réseau où la figure centrale d'Élie Marion peu à peu laisse la place aux adeptes anglais ou écossais. Si bien qu'à la mort du cévenol, en 1713, les «French prophets» sont majoritairement issus du pays d'accueil ${ }^{39}$. Sans doute la nécessité de cette nouvelle configuration était-elle inscrite dès le début de l'aventure "européenne" au principe même des troubles que les inspirés venus à insurrection avaient provoqués dans les rangs des Églises du Refuge. J.-P. Chabrol rappelle les innombrables pamphlets et libelles qui accablèrent de sarcasmes et d'ironies les semeurs de paroles étranges et de prophéties d'accomplissement de la "vérité » et de sa loi. Marion, condamné en 1707 au pilori aux côtés de Jean Daudé et de Nicolas Fatio, est coupable d'une spiritualité en excès, et d'une impossibilité à rigoureusement parler "politique»: comment en effet demeurer impunément en une société que l'on dit scandaleuse aux yeux d'un dieu qu'elle prétend servir? Si les prophètes furent " scandale » au monde, de bien plus ample outrage est le monde qui se scandalise en eux. Et ce « monde » récuse tout ce que peuvent proférer à son encontre les « vagabonds » de Dieu. Fatio fut donc mené au pilori, portant cet écriteau : convaincu « d'avoir encouragé et favorisé Marion dans ses prophéties scélérates et simulées, et de les avoir fait imprimer et publier pour terrifier les sujets de la Reine $»^{40}$.

20 Nous voici au plus près de l'une des questions centrales posées par le prophétisme languedocien, dont l'A. rappelle l'importance dans la constitution de l'«illuminisme» comme vecteur de connaissance à l'aube des Lumières. Nicolas Fatio est un "passant considérable »: géomètre, mathématicien, correspondant de Newton, membre de la Société Royale, il est dans le même temps en passion d'astrologie, d'alchimie, de kabbale. Homme de son temps, à coup sûr : Newton, son protecteur jusqu'au temps des prophètes, ne cessera à son tour d'intriguer ses contemporains et ses lecteurs d'aujourd'hui pour mêler regard de science et investigations aux «marges ». Sa "malle» est désormais célèbre, qui livra il y a peu un étrange composite de manuscrits fondateurs de nouvelle et rigoureuse science, et d'exégèses philosophico-alchimistes ${ }^{41}$. Fatio appartient à ce monde 
métis: ici l'univers de la loi, et ici même, en registre d'égale compétence, ce qu'on pourrait nommer l'analytique de la raison oblique. Dont le prophétisme, par privilège, relève. Au croisement de cette double mouvance, le lieu qui me semble en donner l'argument : l'espace de l'imaginaire, où gît toute disposition au rêve. Des rêves, Fatio en est fréquemment sujet et scripteur : rêveries d'emblèmes bibliques où la croix cède au dragon la place ; songes d'espaces de va-et-vient, de pur transit, de pur passage ; image de bateaux croisant en haute mer, en haut désert ; rêves, enfin, de demeure brisée du monde ${ }^{42}$. Ensemble de forte signifiance : tout obéit, en ces textes, au paradigme de la double expérience qui aboute la prophétie, en tant que "vérité errante» comme chose "de néant $»^{43}$, au parcours de la connaissance en ses bifurcations et incertitudes de principe, jusqu'à ce que l'indécidabilité de l'une et l'autre vaille indice de vérité. C'est en cette zone, obscure en même temps que surexposée, surexplosée, de non-contradiction, - ce « je ne sais quoi » des XVII et XVIII ${ }^{e}$, ce site de l'« inconscient », que le XX ${ }^{e}$ siècle nommera ainsi définitivement -, que se nouent aventure de la connaissance et procès prophétique. Jusqu'à ce que l'un et l'autre, au moins en leurs strates de surface, entrent en divergence et s'autonomisent quant à leurs formules et effets. Mais en ce prime temps de la genèse de la "vérité », il n'est de nuit que partagée, entre passion de science et passion de prophétie. Si bien que Nicolas Fatio n'advient pas au hasard sur la «scène» des inspirations : en sa singularité se conjuguent le réel comme site du rationnel, selon la définition qu'en proposera plus tard Hegel, et le (même) réel comme œuvre à l'innommable, où rien ne se peut "représenter", sinon ce rien, cette pure présence. Ici s'engendre le prophétisme, apte à faire en effet présence au point le plus névralgique de toute historicité, lorsque toute métaphore échoue à dire l'histoire qui se fait ; là s'entame la longue marche du savoir, lorsque la mesure du monde s'impose comme son habitude, cette seconde nature.

Les prophètes languedociens, dont J.-P. Chabrol restitue magistralement avec Élie Marion l'une des figures les plus ardentes, et de plus forte radicalité sans doute, récapitulent - en l'insoumission camisarde dont ils furent pour l'essentiel partie prenante et capitale, leurs énoncés de déréliction et de jugements qui insinuèrent dans le champ religieux calviniste les scandales les plus féconds, et leurs parcours européens en tous sens, - les axes fondateurs de toute inspiration venue au vif d'une histoire saccagée. Si la perte est en effet en amont toujours de la prophétie, si le malheur qui se dit à venir est d'autant plus certain qu'il est déjà au profond de l'histoire advenue, l'inspiration ne peut vivre, et dire, cette histoire, que comme brûlement des siècles, et leur temps accompli. J.-P. Chabrol montre alors avec excellence que là, en cette séquence d'une histoire entre deux morts, les prophètes cévenols devaient former réceptacle et creuset de toutes les passions contemporaines de leur quête. Les "French prophets", ces témoins d'un monde désormais absent, pouvaient ainsi inscrire leur action dans la très longue expérience des illuminations, et à leur tour féconder mille sites en travail de souffrance et de deuil. Pour pleinement comprendre cette intrigue sociale et culturelle insoupçonnée à l'aube des Lumières, J.-P. Chabrol vient de livrer ici un ouvrage essentiel. 


\section{NOTES}

1. Sur le sujet des convulsions, auquel je viendrai en détail plus tard, Jean-Pierre Chantin se réfère à l'ouvrage de Catherine Laurence MAIRE, Les convulsionnaires de Saint-Médard (Paris, Gallimard-Julliard, 1985 [coll. «Archives»]). À s'en tenir à la période la plus récente, d'autres travaux, porteurs de thèses différentes, auraient pu être rappelés : B. Robert KREISER, Miracles, Convulsions and Ecclesial Politics in Eighteenth Century Paris (Princeton, Princeton University Press, 1978); François MABILAT, La divine surprise, les convulsionnaires parisiens et la société du XVIII ${ }^{e}$ siècle, Paris. Thèse de l'E.H.E.S.S., 1982 ; Daniel VIDAL, Miracles et convulsions jansénistes au XVIII siècle-le mal et sa connaissance, Paris, P.U.F., 1987 [cf. Arch. 65.366)). Parmi les " archives à consulter ", citées par l'A., on peut signaler en complément le Fonds Sainte-Beuve, Société d'histoire du Protestantisme, Paris.

2. L'une des présentations les plus précises de cette phase historique est sans conteste celle de Joseph DEDIEU, «L'agonie du jansénisme », Revue d'Histoire de l'Eglise de France, janvier-mars 1928, XIV, pp. 161-214. Cf. aussi Léon SÉCHÉ, Les derniers jansénistes depuis la ruine de Port-Royal jusqu'à nos jours, 1710-1870, 3 vol., Paris, Perrin, 1891.

3. Abbé Joseph DEDIEU, «L'agonie du jansénisme », art.cit.

4. PASCAL, Pensées, 553, « Le mystère de Jésus », Paris, Garnier, 1951, éd. Brunschvicg, p. 210.

5. Il en va ainsi du prophétisme languedocien, et son accomplissement dans et contre le temps, repoussant sans cesse la fin, et dont témoignent les textes d'Élie Marion. J'y viendrai à propos de l'ouvrage novateur de Jean-Paul Chabrol, cf. infra.

6. Jean ORCIBAL, Port-Royal entre le miracle et l'obéissance. Flavie Passart et Angélique de Saint-Jean Arnauld d'Andilly, Paris, Seuil, 1957.

7. Ainsi de François-Philippe Mésenguy (1677-1763), appelant, partisan des thèses quesnellistes, mais hostile à l'œuvre des convulsions, ou Paul Collard (1698-1775), oratorien, familier de François de Pâris, favorable à l'argumentaire miraculiste, mais opposé à la théorie du spasme, ou encore François-Hyacinthe Delan (1672-1754), figure janséniste de premier plan, adversaire du mouvement convulsionnaire.

8. Louis TRÉNARD, Lyon, de l'Encyclopédie au Préromantisme, Paris, P.U.F., 1958, 2 vol. Cf. notamment vol. 1: La philosophie des Lumières, chap. $\mathrm{V}$, où sont présentés les courants mystiques et illuministes qui traversèrent et travaillèrent la cité.

9. Mino bergamo, L'anatomie de l'âme. De François de Sales à Fénelon, Grenoble, J. Millon, 1994 (cf. Arch. 90.4).

10. Innombrables sont les écrits anonymes traitant $\mathrm{du}$ « naturalisme » des convulsions. Cf. par exemple Essais de Physique, où l'on démontre par les règles de la nature, comment se font les convulsions qui attaquent les malades au tombeau de Mr Pâris, et sur le chemin qui y conduit, s.l.n.d., BnF, Ld4 1754 ; Observations de médecine sur la maladie appelée convulsion, par un médecin de la Faculté de Paris, Paris, 1732, 32 p., etc. Mais l'ouvrage capital demeure celui de Philippe HECQUET, proche de Port-Royal où il succéda à Hémon comme médecin, tolérant les premiers miracles au tombeau de Pâris. Opposé aux états du spasme, il dénonce Le naturalisme des convulsions, démontré par la physique, par l'histoire naturelle et par les evenemens de cette cuvre, et démontrant l'impossibilité du Divin qu'on lui attribue dans une lettre sur les secours meurtriers, 2 parties, Soleure, 1733.

11. J'ai rappelé dans mon ouvrage La morte-raison. Isaac la juive, convulsionnaire janséniste de Lyon 1748-1841, (Grenoble, J. Millon, 1994) (cf. Arch. 90.71). la position de Sainte-Beuve. Je la cite à nouveau, pour l'économie de ma démonstration : «Cette idée (...) que Port-Royal, et tout ce qui y 
avait rapport, méritait d'être le théâtre et l'objet manifeste de faveurs surnaturelles, s'entretint continuellement depuis le miracle de la Sainte-Épine, et, redoublant à chaque persécution, contribua fort à exciter enfin le scandale des convulsions. Du sein de la gloire des Provinciales, c'est une perspective fâcheuse qui nous est ouverte. Le mal caduc est au bout » (Port-Royal, Paris, La Pléiade, éd. 1984, t. II, livre III, p. 197).

12. Je me permets de renvoyer à mon ouvrage Miracles et convulsions jansénistes au XVIII ${ }^{e}$ siècle, op. cit., où j'étudie la signification des crucifiements, incisions, secours, abjections, et tout ce qui se dit dans les états de miracle et de convulsion comme raison du rapport social, en même temps que procès de connaissance.

13. Bernard LAMBERT, Idée de l'œuvre des secours selon les sentiments de ses véritables défenseurs, 3 vol. Son ouvrage contre la prestation de serment, Lettres aux ministres de la ci-devant Église constitutionnelle, est publié en 1795-1796.

14. Camille LATREILle, La petite Église de Lyon, H. Lardanchet, 1910.

15. Daniel VIDAL, Miracles et convulsions jansénistes au XVIII ${ }^{e}$, op. cit.

16. Arsenal, ms. 6883.

17. Arsenal, ms. 6889.

18. BnF, ms. 4113.

19. Arsenal, ms. 6882.

20. Ibid.

21. lbid.

22. Anonyme, BnF. ms. 4261, notamment fol. 193-235.

23. Ainsi Dom Deforis, originaire du Forez (1732), convulsionnaire. refusera la prestation de serment et sera guillotiné en 1794 (Bibl. de la Société de Port-Royal, fonds le Paige, Notice Historique I).

24. Cf. Émile APPOLIS, Entre jansénistes et zélantis : le tiers-parti catholique au XVIII ${ }^{e}$ siècle, Paris, A. et J. Picard, 1960.

25. Daniel VIDAL, La morte-raison, op.cit.

26. lbid., chap. III, « Nativités de la parole».

27. Jérôme BEsorgne, Histoire de l'abbaye de Port-Royal, Cologne, Aux dépens de la Compagnie de Jésus, éd. 1752, vol III, p. 219 ss.

28. Philippe JOUTARD, La légende des camisards. Une sensibilité au passé, Paris, Gallimard. 1977 ; Henri BOSC, La guerre des Cévennes, 1702-1710, 6 vol., Montpellier, Presses du Languedoc. 1986-1990; Daniel VIDAL, L'Ablatif absolu, théorie du prophétisme, Paris, Anthropos, 1977 ; Le malheur et son prophète. Inspirés et sectaires en Languedoc calviniste, 1685-1725, Paris, Payot, 1983.

29. Ainsi du recueil des prophéties énoncées par Élie Marion et ses compagnons en 1712-1713, lors de leur voyage en Provinces-Unies, en Europe centrale, et à Rome, qu'Élie n'atteindra pas : Quand vous aurez saccagé vous serez saccagés, car la lumière est apparue dans les ténèbres pour les détruire, Londres, s.l. imprimé par les soins de Nicolas Fatio, 1714.

30. à Rotterdam, A. ACHER, 1686. L'année suivante, paraît l'Apologie pour l'accomplissement. Dès les débuts de l'inspiration cévenole, Pierre JURIEU apporte un soutien décisif aux manifestations de l'esprit. Ses Lettres pastorales adressées aux fidèles de France, A. ACHER, 1688 et son ouvrage plus politique Les soupirs de la France esclave qui aspire après la liberté (1689-1690) témoignent de la ferveur de son engagement aux côtés des inspirés.

31. Édités par Maximilien Misson, Londres, avril 1707.

32. Prophétie du 2 janvier 1707.

33. Prophétie du 14 janvier 1707.

34. L'ouvrage de référence sur les 'French Prophets' est le livre de Hillel schWARTZ, The French Prophets in England : a Social History of Millenarian Group in the Early Eighteenth Century, Londres, Yale University, 1976. 
35. François LAPLANCHE, L'écriture, le sacré et l'histoire. Érudits et politiques protestants devant la Bible en France au XVII ${ }^{e}$ siècle, Amsterdam, Apa-Holland University Press, 1986.

36. Parmi ces mystiques mêlés aux émotions populaires, une femme, Margery KEMPE qui rédige en 1436 son autobiographie, Le Livre. Une mystique anglaise au temps de l'hérésie lollarde (1436), Grenoble, J. Millon, 1987 (trad. et préface : «Margery Kempe ou la dévoration du temps », par Daniel Vidal) (cf. Arch. 70.347).

37. Voltaire, Lettres Philosophiques, 1734, expression que rappelle opportunément Jean-Paul Chabrol. (Cinquième Lettre : Sur la religion anglicane : «C'est ici le pays des sectes. Un Anglais, comme homme libre, va au Ciel par le chemin qui lui plaît..» éd. 1964, Paris, GarnierFlammarion, p. 42). Bien entendu, ce terme ne peut être compris dans l'acception péjorative que nous lui donnons aujourd'hui, mais comme assemblée cultuelle, toute « effervescence " annulée.

38. Cf. le recueil de prophéties énoncées en 1712 par Élie MARION et Jean ALLUT, à Londres, Provinces-Unies et Stockholm : Plan de la Justice de Dieu sur la terre dans les derniers jours, et du relèvement de la chute de l'homme par son péché, éd. 1714.

39. Jean-Paul Chabrol note que dès 1715 , les inspirés français ne représentent plus que le tiers de la « communauté ", dont on peut suivre la trace pendant plusieurs décennies. Elle est alors forte de 500 à 600 membres, la plupart des « frères » venant de la bonne « bourgeoisie citadine ».

40. Cité par Jean-Paul CHABRoL d'après Hillel SCHWARTZ, op.cit.

41. Dans son ouvrage La malle de Newton, Paris, Gallimard, 1993, Loup VERLET commente l'articulation entre ces deux postures, que révèlent les manuscrits de Newton, réapparus en 1936 à l'occasion d'une vente publique. Il signale la relation d'extrême amitié que le maître entretenait avec Nicolas Fatio de Duillier.

42. Papiers Fatio, Ms français 602, fasc. 1, fol. 199-208, Bibl. Publ. Université de Genève. J'ai proposé une analyse de ce travail de rêve in Le malheur et son prophète, op.cit., $2^{\mathrm{e}}$ Partie, chap. III, p. 174 et ss. Jean Allut, compagnon inspiré d'Élie Marion, menuisier et de bonne instruction, à son tour fait des rêves. Celui-ci, par exemple, qu'il nomme "Songe chymique de J.A. », en date du 11 avril 1718, à Worcester, qui débute ainsi : «J'ai songé que j’étois dans un cham au bor d'un fossé et environd au milieu de l'épesseur du bor..", dont j'ai proposé une lecture en termes de castration, de désert et de mort, ibid, pp. 189 ss.

43. «Mon enfant, ma vérité est errante, je te dis : elle ne trouve aucun lieu de retraite sur la Terre...Je suis errant, je te dis, comme les choses de néant ", énonce d'Élie MARION, Avertissements prophétiques, 16 janvier 1707. Par quoi il ne faut pas entendre le seul désastre de la parole de Dieu, mais la condition même du prophétisme, ce désastre recommencé. Cf. Daniel viDAL, L'Ablatif absolu, op.cit.

\section{AUTEUR}

\section{DANIEL VIDAL}

Centre d'Études des Mouvements Sociaux - C.N.R.S. 